\title{
Performance Comparison of Various Diversity Techniques using Matlab Simulation
}

\author{
Sanjiv Kumar \\ Department of Computer Engineering, BPS Mahila Vishwavidyalaya, Khanpur, Kalan-131305, India \\ E-mail: skganghas@rediffmail.com \\ P. K. Gupta \\ Department of Computer Science and Engineering, \\ Jaypee University of Information Technology, Waknaghat, Solan - 173 234, India \\ E-mail: pradeep1976@yahoo.com \\ G. Singh \\ Department of Electronics \& Communication Engineering, \\ Jaypee University of Information Technology, Waknaghat, Solan - 173 234, India \\ E-mail :drghanshyam.singh@yahoo.com \\ D. S. Chauhan \\ Uttarakhand Technical University, Deharadun, India \\ E-mail:drdschauhan@gmail.com
}

\begin{abstract}
Due to the time-varying nature of the wireless channel and presence of limited resources for the signal transmission, which faces various detrimental effects such as path-loss, delay spread, Doppler spread, shadowing and interference make it very difficult to achieve sufficient data rates. To combat the effect of multi-path fading in the wireless communication system, the diversity combining mechanism has been introduced. In this paper, we have developed an algorithm for the performance evaluation of various spatial diversity combing techniques by using Matlab simulation. The developed new algorithm combine all three diversity techniques such as maximal ratio combining (MRC), selection combining (SC) and equal gain combing (EGC) techniques The combined diversity techniques algorith $\mathrm{m}$ computes and compares the MRC, SC, and EGC combing techniques theoretically by using the Matlab simulation.
\end{abstract}

Index Terms - Maximal-Ratio Combining, Selection Combining, Equal-Gain Combining, Fading, Rayleigh Channel

\section{Introduction}

Diversity combining is a potential method by which multiple replicas of the same information carrying signal received over several diversity branches are combined with specific manner in order to combat the diverse effects of the multipath fading in wireless communication systems [1-6]. However, the diversity combining methods have been implemented either at receiver or transmitter, or on both for combating very effectively the effects of multipath fading at a relatively low cost. For instance, let us consider the design of an antenna array receiver for the millimeter-wave communications, where several tens of array elements have been placed on the surface of a portable receiver [7-10]. Thus, there is a need for significant diversity combining techniques to be used effectively with large antenna arrays. However, the suboptimal receiver structures may exploit other statistics or consider a partitioned diversity combining scheme [10] to achieve the performance comparable to the optimal receiver. Then, it would be highly desirable to assess the efficacy of several receiver design options to obtain the most appropriate choice with reference to the comple xity and implementation constraints.

Due to the time-varying nature of the wireless channel and presence of limited resources for transmission, the transmitted signal faces various detrimental effects such as path loss, delay spread, Doppler spread, shadowing and interference which make it very difficult to achieve high data rates. To mitigate the effect of multipath fading in the wireless communication, a most popular mechanis m known as diversity has been introduced. In the telecommunication system, the diversity schemes is a method for improving the reliability of a message signal by using two or more communication channels with different characteristics. It plays significant role in combating the signal fading and co-channel interference without error bursts. 
Alternatively, a redundant forward error correction code can be used. The diversity technique exploits the multipath propagation and provides the diversity gain (measured in decibels) [2-10]. The diversity combining technique can be exploited over time, frequency and space domains. However, it is well known that the diversity reception is an efficient communication receiver technique for mitigating the detrimental effects of multipath fading by increasing the overall signal-tonoise ratio (SNR) and improve the radio link performance in the wireless mobile channels at relatively low cost [11-21].

In this paper, we have developed an algorith $\mathrm{m}$ for the performance evaluation of various spatial diversity combing techniques by using Matlab simulation. The most important diversity reception methods employed in digital communication receivers are maximal ratio combining (MRC), equal gain combining (EGC), and selection combining (SC). However, MRC is the optimal technique in the sense that it attains the highest SNR as compared to any combining scheme as well as independent of the distribution of the branch signals since it results in a maximum-likelihood receiver. The remainder of the paper is organized as follows. Section 2 is concern with the diversity system model. In section 3 , proposed algorithm for the performance evaluation has been developed. Section 4 discusses about the simulation results for various diversity techniques by using developed algorithm. Finally, section 5 concludes the work.

\section{Diversity System Model}

The diversity system model consists of a physical model by assuming the fading to be independent from one element to the next and each element acts as an independent sample of the random fading process (Rayleigh) means each element of the array receives an independent copy of the transmitted signal [9, 11]. In this system model, we emphasized to combine all these independent samples to yield the enhanced signal-tonoise ratio (SNR) and reducing bit-error-rate (BER). However, the diversity works because we receive $N$ independent copies of the same signal at array of $N$ antennas. If at least one copy has sufficient power, one should be able to process the signal. Here, we consider a single-user system model wherein the received signal is a sum of the desired signal and noise [2, 3].

$$
x=h u(t)+n
$$

where $u(\mathrm{t})$ is the unit power signal transmitted, $h$ represents the channel (including the signal power) and $n$ the noise. The power in the signal over a single symbol period, $T_{\mathrm{s}}$, at element $n$, is

$$
\begin{aligned}
P & =\frac{1}{T_{s}} \int_{0}^{T_{s}}\left|h_{n}(t)\right|^{2}|u(t)|^{2} d t \\
& =\left|h_{n}(t)\right|^{2} \frac{1}{T_{s}} \int_{0}^{T_{s}}|u(t)|^{2} d t=\left|h_{n}\right|^{2}
\end{aligned}
$$

Since we are assuming slow fading, the term $|\mathrm{h} n(\mathrm{t})|$ remains constant over a symbol period and can be brought out of the integral and $\mathrm{u}(\mathrm{t})$ is assumed to have unit power. Setting $E\left\{\left|n_{n}(t)\right|^{2}\right\}=\sigma^{2}$ and we get the instantaneous SNR at the $\mathrm{n}^{\text {th }}$ element $\left(\gamma_{n}\right)$ as:

$$
\gamma_{n}=\frac{\left|h_{n}\right|^{2}}{\sigma^{2}}
$$

This instantaneous SNR is a random variable with a specific realization given the channel realization $h_{n}$. The expectation value taken to estimate the noise power is therefore consider over a relatively short time period [2]. We are assuming Rayleigh fading, so $h_{n}=\left|h_{n}\right| e^{j<h_{n}}$ where $\angle h_{n}$ is uniform in $[0,2 \pi]$ and $\left|h_{n}\right|$ has a Rayleigh pdf, implying $\left|h_{n}\right|^{2}$ and $\gamma_{n}$ has an exponential pdf such as [3]:

$$
\begin{aligned}
& \left|h_{n}\right|=\frac{2\left|h_{n}\right|}{P_{0}} e^{-\frac{\left|h_{n}\right|^{2}}{p_{0}}} \\
& \gamma_{n} \cong \frac{1}{T} e^{-\gamma_{n / \Gamma}} \\
& \Gamma=E\left\{\gamma_{n}\right\}=\frac{E\left\{\left.h_{n}\right|^{2}\right\}}{\sigma^{2}}=\frac{P_{0}}{\sigma^{2}}
\end{aligned}
$$

The instantaneous SNR at each element which is an exponentially distributed random variable. $\Gamma$ represents the average SNR at each element. This is also the SNR of a single element antenna that is the SNR if there is no array. Therefore $\Gamma$ serve as a baseline for improvement in the SNR.

\subsection{Selection Combining Diversity}

At the receiver, if we have now $N$ copies of the same transmitted symbol. Then we have to combine them effectively to reliably recover the transmitted data. We consider the fading for each signal is independent. In the selection combining diversity, the receiver selects the antenna with the highest received signal power and ignores observations from the other antennas. We assign weights such that for maximum power signal provide weight 1 and for rest of others weight 0 . Therefore, we are able to get one significant signal at 
the output. For deriving the mathematical expressions for the selection combining diversity, we obtain the set of weights $w$. To obtain the weight vector value, we assume that the receiver has the required knowledge of the channel fading vector $h$. As each element is an independent sample of the fading process, the element with the greatest SNR is chosen for further processing. In the selection combining diversity, the expression instantaneous SNR with weight function can be written as [2-5]:

$$
w_{k}= \begin{cases}1 & \gamma_{k}=\max _{n}\left\{\gamma_{n}\right\} \\ 0 & \text { otherwise }\end{cases}
$$

Since the element chosen is the one with the maximum SNR, the output SNR of the selection diversity scheme is $\gamma=\max _{n}\left\{\gamma_{n}\right\}$. This technique requires only the measurement of the signal power no phase shifters and variable gain. The parameters for analyzing such systems are the outage probability, BER and resulting improvement in SNR. The outage probability provides the probability that the output SNR falls below a threshold value $\gamma_{s}$. This expression is valid only when the fading at each element is assumed independent. By using the pdf of $\gamma_{n}$, then the outage probability is $[2,8]$ :

$$
P_{\text {out }}\left(\gamma_{s}\right)=\left[1-e^{\gamma_{s} / \Gamma}\right]^{N}
$$

From (8), the outage probability decreases exponentially with the number of elements. $P_{\text {out }}$ represents the cdf of the output SNR as a function of threshold $\gamma_{s}$.

\subsection{Maximal Ratio Combining Diversity}

In order to maximize the output SNR of the signal, we cannot choose one signal and neglect others. So we combine the signals on such a way that the output signal provides all transmitted information. In MRC, we assign the weighted bits to the signal in such a way that all the signals are strong, which is performed in the order to improve the faded signals. The branches with strong signals are further amplified and those which are week are attenuated. Then we combine the signals to get output signal, which improved the performance than the selection combining diversity, however it is too complex to implement. Its weighted bit allocation process is complex and we have to know the exact signal at the receiver $[10,16]$. The received signal at the array elements as a vector $\mathrm{x}(\mathrm{t})$, and the output signal as $\mathrm{r}(\mathrm{t})[2,3]:$

$$
x(t)=h(t) u(t)+n(t)
$$

where

$$
\left\{\begin{array}{l}
h=\left[h_{0}, h_{1,} \ldots . h_{N-1}\right]^{T} \\
n=\left[n_{0}, n_{1}, \ldots . . n_{N-1}\right]^{T}
\end{array}\right.
$$

and

$$
r(t)=w^{H} x=w^{H} h u(t)+w^{H} n
$$

Since the signal $u(t)$ has unit average power, the instantaneous output SNR is:

$$
\gamma=\frac{\left|w^{H} h\right|^{2}}{E\left\{\left|w^{H} n\right|^{2}\right\}}
$$

The noise power in the denominator is given by:

$$
\begin{aligned}
& P_{n}=E\left\{\left|w^{H} n\right|^{2}\right\}=E\left\{\left|w^{H} n n^{H} w\right|\right\} \\
& =E\left\{w^{H} E\left\{n n^{H}\right\} w\right\}=\sigma^{2} w^{H} I_{N} w \\
& \sigma^{2} w^{H} w=\sigma^{2}\|w\|^{2}
\end{aligned}
$$

and $I_{N}$ represents the $\mathrm{N} \times \mathrm{N}$ identity matrix. Since the constants do not matter, one could always scale $w$ such that $\|\mathrm{w}\|=1$. The SNR is therefore given by [2]:

$$
\gamma=\frac{\left|w^{H} h^{2}\right|}{\sigma^{2}}
$$

By the Cauchy-Schwarz inequality, it has a maximu $\mathrm{m}$ value when $\mathrm{w}$ is linearly proportional to $h$, or $w=h$, then

$$
\gamma=\frac{\left|w^{H} h^{2}\right|}{\sigma^{2} h^{H} h}=\frac{h^{H} h}{\sigma^{2}}=\sum_{n=0}^{N-1} \frac{\left|h_{n}\right|^{2}}{\sigma^{2}}
$$

or

$$
\gamma=\sum_{n=0}^{N-1} \gamma_{n}
$$

The output SNR is, therefore, the sum of the SNR at each element. However, the better diversity combiner can choose the weights to the fading for each element. In some sense, this answer is expected since the solution is effectively the matched filter for the fading signal. We know that the matched filter is optimal in the single user case. By using (15) expected value of the output SNR is therefore $N$ times the average SNR at each element, which is [3]: 


$$
E\{\gamma\}=N \Gamma
$$

This indicates that on average, the SNR improves by a factor of $N$, which is significantly better than the factor of $(\ln N)$ improvement in the selection combining diversity case. To determine the pdf of the output SNR, we use the fact that the pdf of the sum of $N$ independent random variables is the convolution of the individual pdfs. Further, the convolution of two functions is equivalent to multiplying the two functions in the frequency (or Laplace) domain. We know that each $\gamma_{n}$ in (15) is exponentially distributed. The characteristic function of a random variable $X$ is given by $E\left\{e^{-s X}\right\}$ that is, the characteristic function (the Laplace transform) of the pdf.

$$
\begin{aligned}
& F_{\Gamma_{n}}(s)=E\left\{e^{-s \gamma_{n}}\right\}=\frac{1}{1+s \Gamma} \\
& F_{\Gamma}(s)=\left[\frac{1}{1+s \Gamma}\right]^{N} \\
& \operatorname{PDF}(\gamma)=f_{\Gamma}(\gamma)=L^{-1}\left[F_{\Gamma}(s)\right] \\
& =\frac{1}{2 \Pi j} \int_{c-j \infty}^{c+j \infty} \frac{e^{s \gamma}}{(1+s \Gamma)^{N}} d \gamma
\end{aligned}
$$

or

$$
P D F(\gamma)=\frac{\mathbf{1}}{(N-\mathbf{1}) !} \frac{\gamma^{N-\mathbf{1}}}{\Gamma^{N}} e^{-\gamma / \Gamma}
$$

Using this pdf, the outage probability for a threshold $\gamma_{s}$ is given by [3]:

$$
\begin{aligned}
P_{\text {out }} & =P\left(\gamma<\gamma_{s}\right) \\
& =\int_{0}^{\gamma_{s}} \frac{1}{(N-1) !} \frac{\gamma^{N-1}}{\Gamma^{N}} e^{-\gamma / \Gamma} d \gamma \\
P_{\text {out }} & =1-e^{-\gamma_{s} / \Gamma} \sum_{n=0}^{N-1}\left(\frac{\gamma_{s}}{\Gamma}\right)^{n} \frac{1}{n !}
\end{aligned}
$$

\subsection{Equal Gain Combining Diversity}

In this process, we have assigned the equal weights to the receiver branches which amplify the signals equally. With reference to the performance comparison it is comparable to MRC, however easy to implement [1621]. So it is a better option than the selection combining diversity. We require a technique in which the weights vary with the fading signals and the magnitude of which fluctuate over several $10 \mathrm{~s}$ of $\mathrm{dB}$. In the equal gain combiner, [2]:

$$
\begin{aligned}
& w_{n}=e^{j<h_{n}} \\
& w_{n} * h_{n}=\left|h_{n}\right| \\
& w^{H} h=\sum_{n=0}^{N-1}\left|h_{n}\right|
\end{aligned}
$$

The noise and instantaneous SNR are given by:

$$
\begin{gathered}
P_{n}=w^{H} w \sigma^{2}=N \sigma^{2} \\
\gamma=\frac{\left[\sum_{n=0}^{N-1}\left|h_{n}\right|\right]^{2}}{N \sigma^{2}}
\end{gathered}
$$

Using the fact that $\left|h_{n}\right|$ is Rayleigh distributed, we have:

$$
\begin{aligned}
& E\left(\left|h_{n}\right|\right)=\sqrt{\Pi P_{\mathbf{0}}} \\
& E\left(\left|h_{n}\right|^{2}\right)=P_{\mathbf{0}}
\end{aligned}
$$

By using the SNR defined in (28) together with (29) and (30), we can find the mean SNR as:

$$
\begin{aligned}
E\{\gamma\} & =\frac{E\left\{\left[\sum_{n=0}^{N-1}\left|h_{n}\right|\right]^{2}\right\}}{2 N \sigma^{2}} \\
= & \frac{1}{2 N \sigma^{2}} E\left\{\sum_{n=0}^{N-1} \sum_{m=0}^{N-1}\left|h_{n}\right|\left|h_{m}\right|\right\}
\end{aligned}
$$

After some mathematical manipulation and simplifying (31), we get:

$$
E\{y\}=\left[\mathbf{1}+(N-\mathbf{1}) \frac{\Pi}{\mathbf{4}}\right] \Gamma
$$

This analysis reveals that, despite being significantly simpler to implement, the equal gain combiner results in an improvement in SNR that is comparable to that of the optimal maximal ratio combiner. The SNR of both combiners increases linearly with $N$. 


\section{Proposed Combined Algorithm for Diversity Techniques MRC, SC and, EG}

In this section, we have proposed a combined algorithm for the discussed diversity techniques in the preceding sections like maximal ratio combining, selection combining and equal gain. The proposed algorith m completes its functioning in 12 different steps User has to make the choice $\mathrm{A}, \mathrm{B}$, and $\mathrm{C}$ to implement the discussed diversity technique MRC, SC, and EG, respectively and based on the given choice algorithm computes the various operations which are listed in various steps for each diversity techniques. Finally, algorithm computes and compares the simulated outage probability/BER as well as SNR enhancement with the theoretical results.
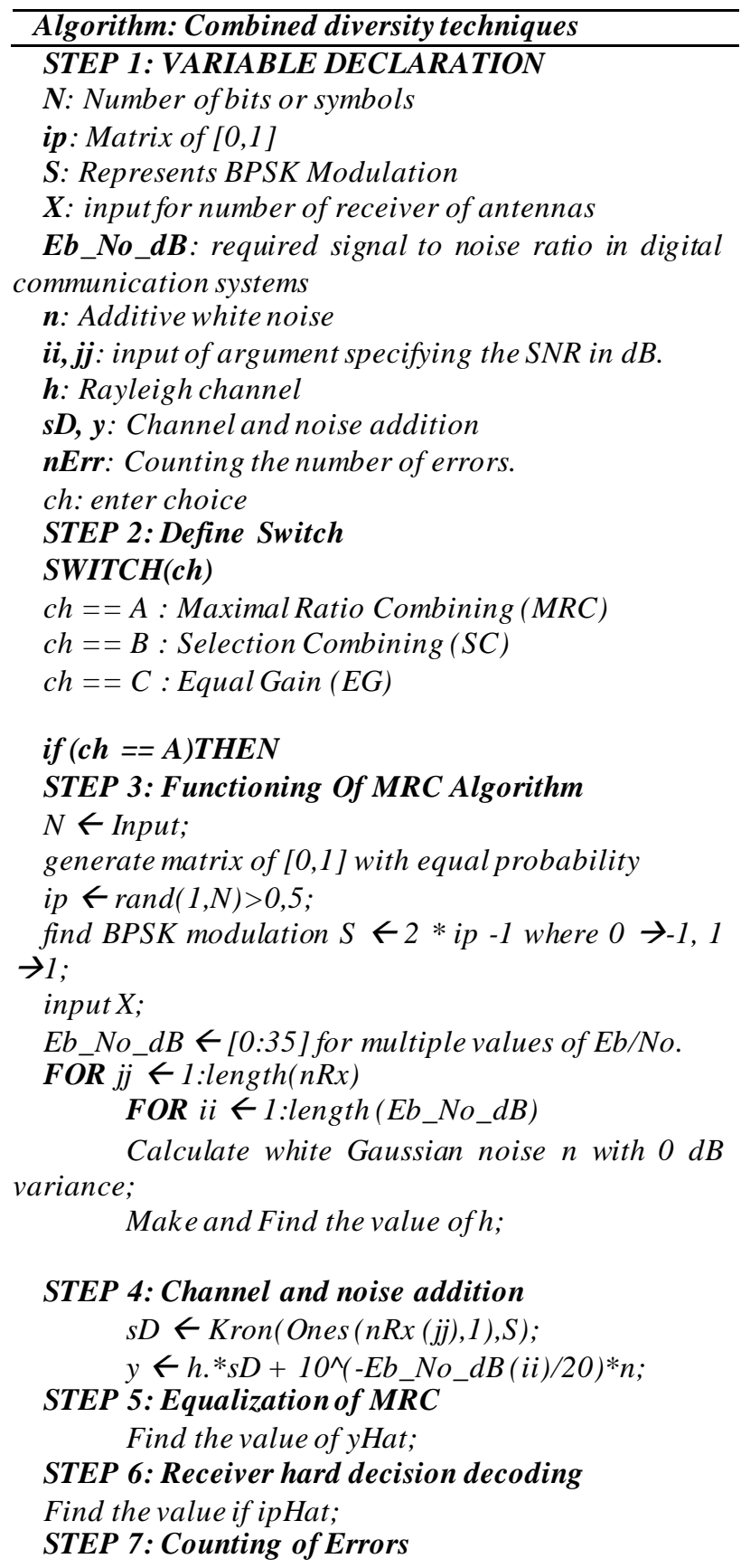

Find the value of $n E r r(j j, i i)$;

END

END

$\operatorname{ELSEIF}(\mathrm{ch}==\mathrm{B})$

STEP 8: Functioning of SC algorithm

REPEAT STEP 3 and STEP 4

STEP 9: Finding the power and max power of channel all rx chain

Find the hPower and hMaxVal of the signal on

STEP 10: Delecting the chain with maximum power Find the value of YSel and hSel;

REPEAT STEP 6 and STEP 7;

ELSE $($ ch $==C)$

STEP 11: Functioning of EG algorithm

REPEAT STEP 3 and STEP 4 ;

STEP 12: Equalization with EQUAL GAIN combining

Find the value of $y \mathrm{Hat}$;

REPEAT STEP 6 and STEP 7;

ENDIF

ENDIF

Calculate and compare simulated BER and theoretical BER;

\section{Simulation Results for Comparison of above Diversity Techniques}

Fig. 1 shows the plot between signal-to-noise ratio and number of received antennas. As we increase the number of received antenna, the SNR is increased significantly. The above plot shows us that the MRC is the best among the three diversity techniques. Equal Gain combining technique is closest to the MRC and the Selection Co mbining is worst among them. In terms of the required processing, the selection combiner is the easiest - it requires only a measurement of SNR at each element, however not the phase or the amplitude. The results presented, used a coherent receiver (the phase of channel is removed after the fact). However, both the maximal ratio and equal gain combiners, on the other hand, require phase information. The maximal ratio combiner requires accurate measurement of the gain too This is clearly difficult to implement, as the dynamic range of a Rayleigh fading signal may be quite large. For this additional cost, for two elements, the MRC improves performance by about $0.6 \mathrm{~dB}$ over the equal gain combiner at a BER of $1 \%$. Table 1 compares the various diversity techniques.

Table 1: Comparison of various diversity techniques

\begin{tabular}{|c|c|c|}
\hline Technique & Circuit Complexity & $\begin{array}{c}\text { C/N Improvement } \\
\text { factor }\end{array}$ \\
\hline $\begin{array}{c}\text { Selection } \\
\text { Diversity }\end{array}$ & N receivers & $1+1 / 2+\ldots+1 / \mathrm{N}$ \\
\hline EGC Diversity & N receivers co-phasing & $1+(\mathrm{N}-1) \pi / 4$ \\
\hline MRC Diversity & $\begin{array}{c}\text { N receivers co-phasing } \\
\text { channel estimator }\end{array}$ & $\mathrm{N}$ \\
\hline
\end{tabular}




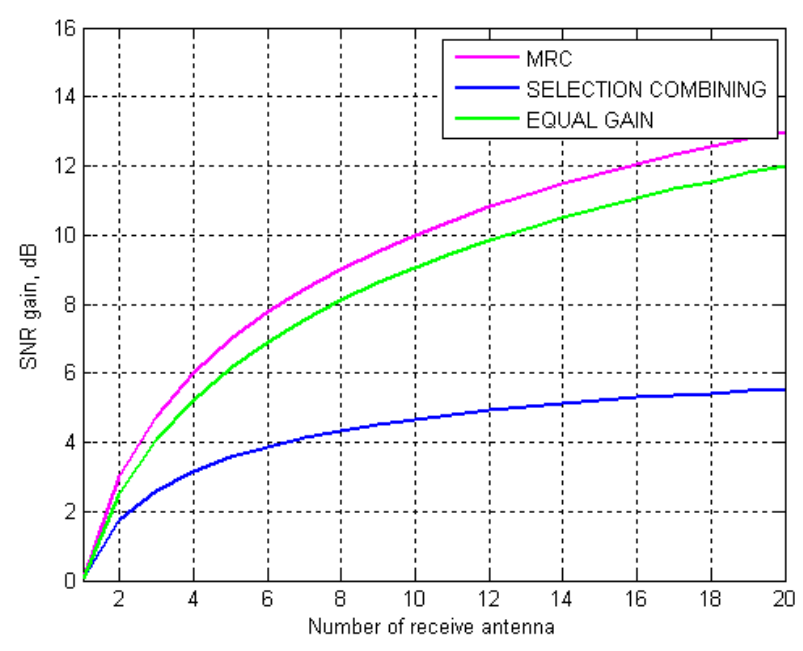

Fig. 1: Comparison of diversity combining techniques at receiver

\section{Conclusion}

The diversity combining techniques are one of the most significant solutions to mitigate the fading problem in the wireless communication systems. It overcomes the effects of flat fading by combining multiple independent fading. However, it entails some penalty in terms of rate, bandwidth, comple xity or size. However, various combining techniques offer different level of complexity and performance. Among all the combining techniques, MRC offer the best performance, however the highest complexity, whereas the selection combining has the lowest performance and least complexity. These techniques are used to improve the performance of radio channel without any increase in the transmitted power. In this paper, we have developed an algorithm for the diversity combining techniques in MATLAB for the comparative analysis. We observed their performance by varying the number of receiver antennas and measuring the SNR at the receivers, which reveals that MRC is the best diversity among all three and selection combing is the worst. We can also observe that the equal gain is also close to MRC in terms of the performance. To compare MRC with selection combining, we have to increase the number of receivers to 4 which will result into a difference of 2.5 $\mathrm{dB}$ in SNR. This difference widens if we further increase the number of antennas at receiver. $\mathrm{MRC}$ is considered better than equal gain as it differs by a gain of $0.5 \mathrm{~dB}$. The output curve steeply rises initially but then becomes smooth. It might be because if we go on increasing the number of receiver antennas the complexities in obtaining the desired signal will also increase.

\section{Acknowledgement}

The authors are very much thankful to the potential reviewers for their critical comments and suggestions to improve the quality of the manuscript.

\section{References}

[1] D. Brennan, "Linear diversity combining techniques," Proc. IEEE, vol. 47, pp. 1075 - 1102, June 1959.

[2] R. Janaswamy, "Radiowave Propagation and Smart Antennas for Wireless Communications," Kluwer Academic Publishers, 2000.

[3] L. C. Godara, "Handbook of Antennas for Wireless Communications", CRC Press, 2002.

[4] S. Verdu, "Multiuser Detection", Cambridge University Press, 1998.

[5] Vahid Tarokh, "New Directions in Wireless Communications Research," Springer Science, New York, 2009.

[6] Andrea Goldsmith, "Wireless Communications," Cambridge University Press, New York, 2005.

[7] H. Zhang and T. A. Gulliver, "Error probability for maximum ratio combining multichannel reception of M-ary coherent systems over flat Rician fading channels," Proc. of IEEE Wireless Communications and Networking Conf., March 2004, vol. 1, pp. 306-310.

[8] Vivek K Dwivedi and G Singh, "Error-rate analysis of OFDM communication system in correlated Nakagami- $m$ fading channel using maximal ratio combining diversity", International Journal of Microwave and Wireless Technologies, vol. 3, no. 6, pp. 717-726, Dec. 2011.

[9] M. S. Patterh, T. S. Kamal and B. S. Sohi, "Performance of coherent square MQAM with Lth order diversity in Rician fading environment," Proc. of $54^{\text {th }}$ IEEE Vehicular Tech., 2001, vol. 1, pp. 141-143.

[10] S. Seo, C. Lee, and S. Kang, "Exact performance analysis of M-ary QAM with MRC diversity in Rician fading channels," Electronics Letters, vol. 40, no. 8, pp. 485-486, April 2004.

[11] M. S. Patterh and T. S. Kamal,"Performance of coherent square M-QAM with Lth order diversity in Nakagami-m fading environment," Proc. of $52^{\text {nd }}$ IEEE Vehicular Tech., 2000, vol. 6, pp. 2849-2853.

[12] G. Fedele, I. Izzo and M. Tanda,"Dual diversity reception of M-ary DPSK signals over Nakagami fading channels," Proc. IEEE International Symp. Personol Indoor, and Mobile Radio Commun., Toronto Ontario, Canada, Sept. 1995, pp. 1195 1201.

[13] I. AI Falujah and V. K. Prabhu, "Performance analys is of MQAM with MRC over Nakagami-m fading channels," Proc. IEEE Wireless Communication and Networking Conf., April 2006, vol. 3, pp. 1332-1337. 
[14] F. J. Altman and W. Sichak, "A simplified diversity Communication system for beyond the horizon links," IRE Trans. on Commun. Systems and Techniques, vol. 4, pp. 50-55, March 1956.

[15] A. Annamalai, C. Tellambura and V. Bhargava, "Equal gain diversity receiver performance in wireless channels," IEEE Trans.on Communications, vol. 48, pp. 1732-1745, Oct. 2000.

[16] A. Annamalai, C. Tellambura and V. Bhargava, "Exact evaluation of maximal ratio and equal-gain diversity receivers for M-ary Qam on Nakagami fading channels," IEEE Trans. on Commun., vol. 47, no. 9, pp. 1335-1344, 1999.

[17] A. Annamalai, C. Tellambura and V. Bhargava, "Unified analysis of equal gain diversity on Rician ana Nakagami fading channels," Proc. IEEE Wireless Communication and Networking Conf., 1999, pp. 10-14.

[18] N. C. Sagias, G. K. Karagiannid is, P. T. Mathiopoulos, and T. A. Tsiftsis, "On the performance analysis of equal-gain diversity receivers over generalized Gamma fading channels," IEEE Trans. on Wireless Commun., vol. 5, no. 10, pp. 2967-2975, Oct. 2006.

[19] G. K. Karagiannid is, "Moments-based approach to the performance analysis of equal gain diversity in Nakagami- $m$ fading," IEEE Trans. on Commun., vol. 52, no. 5, pp. 685-690, May 2004.

[20] Sanjiv Kumar, P. K. Gupta, G. Singh, D. S. Chauhan,"Performance Analysis of Rayleigh and Rician Fading Channel Models using Matlab Simulation", IJISA, vol.5, no.9, pp.94-102, 2013. DOI: $10.5815 /$ ijisa.2013.09.11

[21] Kuwar Pratap Singh, P K Gupta and G Singh. "Performance Evaluation of Enhanced Interior Gateway Routing Protocol in IPv6 Network," International Journal of Computer Applications 70(5):42-47, May 2013. DOI: $10.5120 / 11962-7802$

\section{Authors' Profiles}

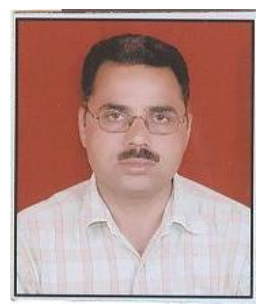

Sanjiv Kumar was born in Kurukshetra, in 1975. He received his B. E. in Electronics and Communication Engineering from the Institution of Engineers (India), Calcutta and M. E. in Electronics and Communication Engineering from Maharishi Dayanand University, Rohtak (Haryana). He is working as a lecturer in the Department of Computer Engineering at B. P. S. Mahila Vishwavidyalaya, Khanpur Kalan (Haryana), India. Currently, he is pursuing his $\mathrm{Ph} . \mathrm{D}$. from Uttarakhand Technical University, Dehradun. His research interests include wireless communication, fading channel models.

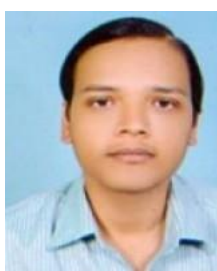

P. K. Gupta received $\mathrm{Ph} \mathrm{D}$ degree in Computer Science and Engineering from the Jaypee University of Information Technology, Waknaghat, Solan, India in 2012. He graduated in Informatics and Computer Engineering from Vladimir State University, Vladimir, Russia, in 1999 and received his M.E. degree in Informatics and Computer Engineering in 2001 from the same university. He has been associated with academics more than ten years in different institutions like BIT M.Nagar, RKGIT Ghaziabad in India. Currently, he is working as Senior Assistant Professor with the Department of Computer Science and Engineering \& IT, Jaypee University of Information Technology, Waknaghat, Solan, India. He has supervised a number of B.Tech/M.Tech/M.Phil. theses from various universities of India. His research interests include Storage Networks, Green Computing, Software Testing and Cloud Computing. He is a Member of IEEE, Life Member of CSI and Life member of Indian Science Congress Association.

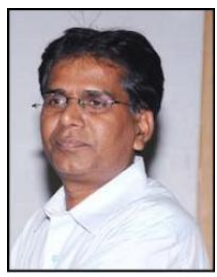

G. Singh: received $\mathrm{Ph} \mathrm{D}$ degree in electronics engineering from the Institute of Technology, Banaras Hindu University, Varanasi, India, in 2000. He was as sociated with Central Electronics Engineering Research Institute, Pilani, and Institute for Plas ma Research, Gandhinagar, India, respectively, where he was Research Scientist. He was also worked as an Assistant Professor at Electronics and Communication Engineering Department, Nirma University of Science and Technology, Ahmedanad, India. He was a Visiting Researcher at the Seoul National University, Seoul, S. Korea. At present, he is Professor with the Department of Electronics and Communication Engineering, Jaypee University of Information Technology, Wakanaghat, Solan, India. He is an author and co-author of more than 170 scientific papers of the refereed Journal and International/National Conferences. His research interests include relativistic electronics, surfaceplasmons, Electromagnetics and its applications, nanophotonics, microwave/THz antennas and its potential applications.

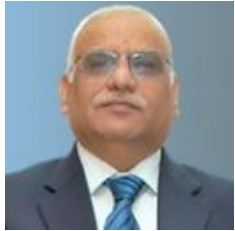

Prof. D. S. Chauhan was born in 1949 at Dholpur, Rajasthan and his parents belong to Mainpuri, Uttar Pradesh. His education took place in Rajasthan, Madhya Pradesh, Uttar Pradesh, Tamilnadu and New Delhi. 
He did his post doctoral work at Goddard Space Flight Centre, Greenbelf Mary land. USA (1988- 91). He did his B.Sc Engg.(1972) in electrical engineering at I.T. B.H.U., M.E. (1978) at R.E.C. Tiruchirapalli (Madras University) and Ph.D. (1986) at IIT/Delhi. His brilliant career brought him to teaching profession at Banaras Hindu University where he was Lecturer, Reader and then has been Professor till today. He has been director KNIT sultanpur in 1999-2000 and founder vice Chancellor of U.P.Tech. University (2000- 2003-2006). Later on, he has served as Vice-Chancellor of Lovely Profession University (2006-07) and Jaypee University of Information Technology (2007-2009) Currently he has been serving as Vice- Chancellor of Uttarakhand Technical University for (2009-till) Tenure. He has been member, NBA-executive AICTE, (2001-04)NABL-DST executive (2002-05) and member, National expert Committee for IIT-NIT research grants.He was Member, University Grant Commission (2006-09). He has been member, CAPART, National executive and chairman central zone, Lucknow from (2001-2004). He has been nominated by UGC as chairman of Advisory committees of four medical universities. Dr Chauhan got best Engineer honour of institution of Engineer in 2001 at Lucknow.

How to cite this paper: Sanjiv Kumar, P. K. Gupta, G. Singh, D. S. Chauhan,"Performance Comparison of Various Diversity Techniques using Matlab Simulation", International Journal of Information Technology and Computer Science(IJITCS), vol.5, no.11, pp.54-61, 2013. DOI: 10.5815/ijitcs.2013.11.06 